\title{
Investigating resonant low-energy electron attachment to formamide: Dynamics of model peptide bond dissociation and other fragmentation channels
}

\author{
Guglielmo Panelli $\odot,{ }^{1}$ Ali Moradmand, ${ }^{2}$ Brandon Griffin, ${ }^{1}$ Kyle Swanson, ${ }^{1}$ Thorsten Weber, ${ }^{3}$ Thomas N. Rescigno, ${ }^{3}$ \\ C. William McCurdy, ${ }^{3,4}$ Daniel S. Slaughter $\odot,{ }^{3, *}$ and Joshua B. Williams ${ }^{1, \dagger}$ \\ ${ }^{1}$ Department of Physics, University of Nevada, Reno, Nevada 89557, USA \\ ${ }^{2}$ Department of Sciences and Mathematics, California Maritime Academy, Vallejo, California 94590, USA \\ ${ }^{3}$ Chemical Sciences Division, Lawrence Berkeley National Laboratory, Berkeley, California 94720, USA \\ ${ }^{4}$ Department of Chemistry, University of California, Davis, California 95616, USA
}

(Received 30 June 2020; accepted 17 August 2020; published 27 January 2021)

\begin{abstract}
We report experimental results on three-dimensional momentum-imaging measurements of anions generated via dissociative electron attachment to gaseous formamide. From the momentum images, we analyze the angular and kinetic-energy distributions for $\mathrm{NH}_{2}{ }^{-}, \mathrm{O}^{-}$, and $\mathrm{H}^{-}$fragments and discuss the possible electron attachment and dissociation mechanisms for multiple resonances for two ranges of incident electron energies, from 5.3 to $6.8 \mathrm{eV}$ and from 10.0 to $11.5 \mathrm{eV}$. Ab initio theoretical results for the angular distributions of the $\mathrm{NH}_{2}{ }^{-}$anion for $\sim 6-\mathrm{eV}$ incident electrons, when compared with the experimental results, strongly suggest that one of the two resonances producing this fragment is a ${ }^{2} \mathrm{~A}^{\prime \prime}$ Feshbach resonance.
\end{abstract}

DOI: 10.1103/PhysRevResearch.3.013082

\section{INTRODUCTION}

The electron-molecule collision process in which a molecule captures a low-energy electron (i.e., with energy up to $\sim 20 \mathrm{eV}$ ), forms a short-lived, unstable molecular anion, and thereafter dissociates into several fragments (one negative ion and all others neutral) is a long-studied process known as dissociative electron attachment (DEA). DEA is among the fundamental electron-molecule collision-based interactions [1-8] and has been found to play an important role in a variety of fields from condensed matter [9-12] and gaseous electronics [13] to low-energy plasmas [14]. The low-energy electrons involved in DEA to molecules in natural settings are typically produced as by-products of primary interactions between matter and high-energy photons or particles. It has been shown that these electrons play a pivotal role in biological processes such as the triggering of DNA strand breaking and other DNA dissociation processes [15-18] and radiation damage of proteins [19].

Formamide $\left(\mathrm{HCONH}_{2}\right)$ is widely considered an archetypal model molecule for the investigation of protein and peptide chemistry due to its simple yet rich structure, which includes an amide bond. The decomposition of formamide into other notable simple organic molecules (e.g., $\mathrm{CH}, \mathrm{HCN}, \mathrm{HCNO}$, etc.) has been widely studied in experimental and theoretical

\footnotetext{
*Corresponding author: dsslaughter@lbl.gov

${ }^{\dagger}$ Corresponding author: jbwilliams@unr.edu
}

Published by the American Physical Society under the terms of the Creative Commons Attribution 4.0 International license. Further distribution of this work must maintain attribution to the author(s) and the published article's title, journal citation, and DOI. settings. Formamide is composed of many of the progenitors of complex biological molecules such as proteins and nucleic acids and is considered an important link in the evolution of simple biomolecules into complex structures. Moreover, formamide has received ample interest due to its $\mathrm{N}-\mathrm{C}$ amide bond. This feature makes formamide a prototypical molecule for the study of electron-capture-induced peptide bond breaking. Investigation of low-energy electron disruption of peptide bonds- the links between amino acids in proteins-is necessary for a more complete understanding of protein stability.

Electron scattering from formamide has been extensively studied previously over a broad range of energies [20-23]. Studies of the dissociation pathways of formamide irradiated by (vacuum) ultraviolet light [24,25] or higher-energy radiation [26-28] have revealed chemical products of biological and technological relevance. Several of these reactions are expected to involve dissociative attachment by low-energy secondary electrons. DEA to gaseous formamide has been studied previously, both theoretically [29-31] and experimentally [31-33]. Thus far, experimental probes of DEA to formamide have focused on fragment yields [32]. Anion fragment momentum imaging provides further details on the dissociation dynamics for fragments resulting from DEA to formamide. The work of Hamann et al. [32] investigated DEA to formamide in the energy range of $0-18 \mathrm{eV}$, and the authors have identified several predominant resonances, of which we probe the major resonances between 5.3 and $6.8 \mathrm{eV}$ and between 10.0 and $11.5 \mathrm{eV}$.

Here, we utilize three-dimensional (3D) ion momentum imaging with an effusive gas target to examine DEA to formamide. Our focus is on the production of $\mathrm{NH}_{2}{ }^{-}, \mathrm{O}^{-}$, and $\mathrm{H}^{-}$. All previous work indicates that the $\mathrm{NH}_{2}{ }^{-}$yield peak between 5 and $7 \mathrm{eV}$ consists of two superimposed resonance bands centered at 5.9 and $6.8 \mathrm{eV}$ [31,32]. Furthermore, the 
work of Li et al. [31] suggests that one of these bands is due to a core-excited dipole-supported resonance formed by low-energy electrons with incident energy within 5-7 eV. The recent Comment of Fedor [34] on that work argues that the more common mechanism of involving doubly excited Feshbach resonances should not be ruled out. In this paper, we investigate whether the angular dissociation distributions and fragment kinetic-energy distributions of $\mathrm{NH}_{2}{ }^{-}$exhibit disparities in the 5-7-eV incident electron energy range that may allow a more direct identification of the resonances responsible for DEA. To aid in the analysis, we employ ab initio theory to identify doubly excited states in the 5.3-6.8-eV energy range that might lead to $\mathrm{NH}_{2}{ }^{-}$production and carry out scattering calculations to determine the expected ion angular distributions, which are compared with measured distributions to confirm the resonant states and product assignments. In addition to an investigation of $\mathrm{NH}_{2}{ }^{-}$production, this work probes the production of $\mathrm{O}^{-}$and $\mathrm{H}^{-}$via DEA to formamide from incident electrons with energy between 10.0 and $11.5 \mathrm{eV}$. We provide feasible production mechanisms along with the results of 3D momentum imaging.

The structure of this paper is as follows. We first provide a description of the experimental apparatus in Sec. II A, followed by an overview of the off-line analysis procedure in Sec. II B. In Sec. III, we give a description of the theoretical methods employed. In Sec. IV we propose electron attachment mechanisms, summarize our results for each fragment of interest, and provide the momentum images, kinetic-energyrelease estimates, and angular dissociation distributions as well as a brief discussion of the results. Lastly, Sec. V contains concluding remarks regarding our results and future directions.

\section{EXPERIMENT}

\section{A. Apparatus}

Details on the experimental apparatus can be found in Ref. [35]. Here, we provide a brief discussion of the most relevant components and specifications. The creation of an effusive formamide molecular target was achieved by a 20-mm-long, 0.3-mm-diameter capillary. We limited condensation of the target gas by precisely controlling the temperature of the liquid reservoir $\left(70^{\circ} \mathrm{C}\right)$, gas tubing $\left(87^{\circ} \mathrm{C}\right)$, and capillary $\left(111^{\circ} \mathrm{C}\right)$. The limitation of residual water in the interaction region was achieved with the use of a liquid-nitrogen cold trap. The background vacuum, without formamide, was below $1 \times 10^{-8}$ Torr, and during operation we achieved a vacuum level of better than $1 \times 10^{-6}$ Torr.

A pulsed electron beam was generated by a commercially acquired electron gun and was directed perpendicularly in relation to the capillary, which produces the molecular target. The device produced 80-ns pulses (including the $\sim 10$-ns rise and fall times) at $50-\mathrm{kHz}$ repetition rate with a full width at half maximum (FWHM) of $0.8 \mathrm{eV}$ for the electron energy distribution. The electron-beam pulse is collimated into a $\sim 1$ mm-diameter region in a uniform magnetic field of $\sim 25 \mathrm{G}$ produced by a pair of 0.75 -m-diameter Helmholtz coils.

After a delay (90-200 ns, depending on the electron-beam energy), the electron pulses were followed by a pulsed elec- tric field in the extraction region of the spectrometer. The spectrometer extracts anions from the electron-molecule interaction region in the direction perpendicular to the electron beam and parallel to the gas jet. Anions produced by DEA were accelerated towards a time-sensitive 80-mm multichannel plate (MCP) detector equipped with a position-sensitive delay-line anode. The time between the electron pulse and ion fragment contact with the MCP detector was recorded together with the position data in list-mode format, allowing for a thorough off-line analysis including cleaning and calibrating. The initial momentum of each ion fragment was determined entirely from the detector position and timing data using the equations of motion in the known electrostatic field.

\section{B. Analysis technique and calibration}

The anion fragment initial momentum data were generated through off-line analysis. The fragment dissociation direction and kinetic-energy distributions were determined from the measured time and position coordinates. Since all anions of interest in this experiment are of the same charge, anions of different mass are distinguished by their recorded time of flight to the detector, which is proportional to the square root of the singly charged anion mass. The time of flight also encodes one coordinate of momentum, limiting the mass resolution of the system. This technique does not allow $\mathrm{O}^{-}$and $\mathrm{NH}_{2}{ }^{-}$to be distinguished by their time of flight; therefore we rely on previous work [32] to identify the dominant fragmentation channel at each incident electron energy. Momentum calibration was performed by measurements of $\mathrm{H}^{-}$and $\mathrm{O}^{-}$ anions from DEA to $\mathrm{H}_{2} \mathrm{O}[13,35,36]$.

For each anion fragment of interest we determine the ion dissociation distribution in 3D momentum space. To display the distribution on two axes with a constant solid angle, we display a conical slice of the 3D momentum sphere. The kinetic-energy and angular distributions are derived from the unsliced momentum. Note that in the analysis we exploited the symmetry about the electron-beam direction axis for the electron attachment and subsequent anion dissociation dynamics. Additionally, the error bars shown in this paper represent one standard deviation in the Poisson statistical uncertainty.

\section{THEORY}

The geometry of ground-state formamide is planar and has $\mathrm{C}_{s}$ symmetry. It is nominally described by the configuration $\left(1-9 a^{\prime}\right)^{2}\left(1 a^{\prime \prime}\right)^{2}\left(10 a^{\prime}\right)^{2}\left(2 a^{\prime \prime}\right)^{2}$. Doubly excited (Feshbach) resonance states can be formed when a colliding electron promotes a target electron in an occupied orbital to an unoccupied orbital and the colliding electron is captured into the same orbital. The lowest unoccupied molecular orbital (LUMO) of formamide is $3 a^{\prime \prime}$, which we denote here as $\pi^{*}$. This is a compact and antibonding valence orbital, which is responsible for the shape resonance seen in low-energy elastic scattering near $2.5 \mathrm{eV}$. By analogy with other systems we have studied, including our recent study of DEA to formic acid [37], we would not expect the low-lying electronic states involving excitation into the $\pi^{*}$ orbital to serve as parents of a doubly excited Feshbach resonance, which are more likely to involve 
TABLE I. Formamide target states and energies used in scattering calculations; experimental energies are from Ref. [20].

\begin{tabular}{lclc}
\hline \hline & & \multicolumn{2}{c}{ Energy $(\mathrm{eV})$} \\
\cline { 3 - 4 } State & Principal configuration & Theor. & Expt. \\
\hline $1^{1} \mathrm{~A}^{\prime}$ & $\left(1 \mathrm{a}^{\prime \prime}\right)^{2}\left(10 \mathrm{a}^{\prime}\right)^{2}\left(2 \mathrm{a}^{\prime \prime}\right)^{2}$ & 0.0 & \\
$1^{3} \mathrm{~A}^{\prime \prime}$ & $\left(1 \mathrm{a}^{\prime \prime}\right)^{2}\left(10 \mathrm{a}^{\prime}\right)^{1}\left(2 \mathrm{a}^{\prime \prime}\right)^{2}\left(3 \mathrm{a}^{\prime \prime}\right)^{1}$ & 4.89 & $\sim 6$ \\
$1^{1} \mathrm{~A}^{\prime \prime}$ & $\left(1 \mathrm{a}^{\prime \prime}\right)^{2}\left(10 \mathrm{a}^{\prime}\right)^{1}\left(2 \mathrm{a}^{\prime \prime}\right)^{2}\left(3 \mathrm{a}^{\prime \prime}\right)^{1}$ & 5.18 & 5.82 \\
$1^{3} \mathrm{~A}^{\prime}$ & $\left(1 \mathrm{a}^{\prime \prime}\right)^{2}\left(10 \mathrm{a}^{\prime}\right)^{2}\left(2 \mathrm{a}^{\prime \prime}\right)^{1}\left(3 \mathrm{a}^{\prime \prime}\right)^{1}$ & 5.46 & 5.2 \\
$2^{3} \mathrm{~A}^{\prime \prime}$ & $\left(1 \mathrm{a}^{\prime \prime}\right)^{2}\left(10 \mathrm{a}^{\prime}\right)^{2}\left(2 \mathrm{a}^{\prime \prime}\right)^{1}\left(11 \mathrm{a}^{\prime}\right)^{1}$ & 5.74 & \\
\hline
\end{tabular}

double occupancy of a $\sigma^{*}$ orbital with substantial Rydberg character.

We employ standard electronic structure methods to compute the energies of the relevant neutral and anion states. Some care is needed to obtain a balanced description of a negative-ion resonance relative to its parent neutral state, which can be sensitive to the choice of molecular orbitals employed. We have found that state-averaged multiconfiguration self-consistent-field (MCSCF) orbitals based on the (triplet) excited neutral states, which are parents of the resonance anion states, form a good basis for characterizing the resonances as well as the excited target states. The orbital basis for the target states and scattering calculations was obtained from a state-averaged, complete active space MCSCF calculation on the neutral target, averaging over the four lowest triplet states. We used a triple-zeta basis of Gaussian functions, augmented with an additional $s$ - and $p$-type function on the oxygen, carbon, and nitrogen atoms. Nine orbitals were constrained to double occupancy, and the remaining six electrons were distributed over an active space of three $\mathrm{a}^{\prime}$ and three $\mathrm{a}^{\prime \prime}$ orbitals, which results in 182 configurations for the target states in both $\mathrm{A}^{\prime}$ and $\mathrm{A}^{\prime \prime}$ symmetry. The energies of the five lowest target states are listed in Table I.

The trial wave function for the scattering calculations used here takes the form [38]

$$
\begin{aligned}
\Psi_{\Gamma_{o} l_{o} m_{o}}^{-} & =\sum_{\Gamma} \hat{A}\left(\chi_{\Gamma} F_{\Gamma \Gamma_{o}}^{-}\right)+\sum_{i} d_{i}^{\Gamma_{o}} \Theta_{i} \\
& \equiv P \Psi+Q \Psi .
\end{aligned}
$$

The first sum contains the direct product of the five $N$-electron neutral target states $\chi_{\Gamma}$ described above and corresponding continuum orbitals $F_{\Gamma \Gamma_{0}}^{-}$, and the second sum runs over $(N+1)$-electron configuration-state functions (CSFs) $\Theta_{i}$, constructed from bound molecular orbitals. The operator $\hat{A}$ antisymmetrizes the product of continuum and target wave functions. The functions $\Theta_{i}$ included in the second sum are of two types. The first type consists of all CSFs that can be constructed consistent with symmetry from the molecular orbitals used to expand the target state functions. This group of CSFs is necessary to relax strong orthogonality constraints between target and continuum functions and to describe short-range correlation effects. The second group of functions $\Theta_{i}$ includes the complement of $P$ space, i.e., the products of virtual molecular orbitals and the remaining target states $(182-5)$ that are energetically closed. This group of terms is essential in describing target relaxation in the presence of an additional electron. Without such terms the resonance state can appear above rather than below its parent neutral state and thereby incorrectly appear to be a core-excited shape resonance instead of a narrow Feshbach resonance.

Resonance parameters are obtained from multistate closecoupling calculations using the well-established complex Kohn method, which has been described previously [38]. The eigenphase sums are fit to a Breit-Wigner form. We use the computed body-frame $S$-matrix elements to connect the theoretical results to laboratory-frame angular distributions by computing the so-called entrance amplitude, which is a complex-valued matrix element of the electronic Hamiltonian between the resonance wave function and a background scattering wave function:

$$
V(\theta, \phi ; \Xi)=\left\langle Q \Psi\left|H_{e l}\right| P \Psi\right\rangle,
$$

where $\theta$ and $\phi$ are the polar and azimuthal angles of the electron momentum vector incident on the fixed-in-space molecular target and $\Xi$ labels the internal nuclear coordinates of the molecule. When the relative orientation of the fragments is not observed, as is the case here, the angular distribution of the DEA product ions is given by

$$
\frac{d \sigma_{D E A}}{d \theta} \propto \int d \phi|V(\theta, \phi ; \Xi)|^{2} .
$$

The procedures we use to construct the entrance amplitudes from the fitted $S$-matrix elements have been described in detail elsewhere [37,39] and will not be repeated here.

Our calculations reveal two Feshbach resonances at 5.52 and $5.65 \mathrm{eV}$ of ${ }^{2} \mathrm{~A}^{\prime \prime}$ and ${ }^{2} \mathrm{~A}^{\prime}$ symmetry, respectively, whose principal configurations are $(\cdots)\left(2 \mathrm{a}^{\prime \prime}\right)^{1}\left(\sigma^{*}\right)^{2}$ and $(\cdots)\left(10 \mathrm{a}^{\prime}\right)^{1}\left(\sigma^{*}\right)^{2}$. The computed entrance amplitudes for these resonances give an expected $\mathrm{NH}_{2}{ }^{-}$angular distribution that is compared with the measured distributions below.

\section{RESULTS AND DISCUSSION}

\section{A. $\mathrm{NH}_{2}{ }^{-}$resonances at $5.3-6.8 \mathrm{eV}$}

We rely on the high-mass-resolution results from Ref. [32], which are able to distinguish between $\mathrm{NH}_{2}{ }^{-}$(mass $16.019 \mathrm{u}$ ) and $\mathrm{O}^{-}$(mass $15.995 \mathrm{u}$ ). This measurement shows that the two fragments occurred at different incident electron energies. The incident electron energies between 5.3 and $6.8 \mathrm{eV}$ are predominantly $\mathrm{NH}_{2}{ }^{-}$, while $\mathrm{O}^{-}$is dominant between 10.0 and $11.5 \mathrm{eV}$.

a. Pathways to formation. Here, we provide plausible formation mechanisms for $\mathrm{NH}_{2}^{-}$and predict the energy threshold for each mechanism (the same is done for $\mathrm{H}^{-}$and $\mathrm{O}^{-}$in the following sections). In doing so, we rely on literature values for standard heat of formation $\Delta H_{f}^{0}$, electron affinity $E_{A}$, and bond dissociation energy $D$ for each fragment and bond involved in the processes considered here. These values, along with the corresponding references, are provided in Table II.

One candidate mechanism for the production of $\mathrm{NH}_{2}^{-}$ from DEA to formamide is through the simple cleaving of the $\mathrm{C}-\mathrm{N}$ amide bond:

$$
e^{-}+\mathrm{HCONH}_{2} \rightarrow\left(\mathrm{HCONH}_{2}\right)^{*-} \rightarrow \mathrm{HCO}+\mathrm{NH}_{2}{ }^{-} .
$$


TABLE II. Summary of thermodynamic data for formamide fragments and bonds relevant to this work.

\begin{tabular}{lcc}
\hline \hline Compound & $\Delta H_{f}^{0}(\mathrm{eV})$ & Electron affinity (eV) \\
\hline $\mathrm{HCONH}_{2}$ & $-1.93[40]$ & \\
$\mathrm{H}_{2} \mathrm{CNH}$ & $1.14[41]$ & \\
$\mathrm{CH}_{3} \mathrm{~N}$ & $3.27[42]$ & \\
$\mathrm{HCN}$ & $1.4[43]$ & \\
$\mathrm{CNH}$ & $1.99[42]$ & \\
$\mathrm{CO}$ & $-1.15[43]$ & $0.77[44]$ \\
$\mathrm{NH}_{2}$ & $1.97[43]$ & $0.75[45]$ \\
$\mathrm{H}$ & $2.26[43]$ & $1.46[47]$ \\
$\mathrm{O}$ & $2.56[46]$ & \\
$\mathrm{Bond}(\mathrm{compound})$ & $D(\mathrm{eV})$ & \\
\hline $\mathrm{C}-\mathrm{N}\left(\mathrm{HCONH}_{2}\right)$ & $4.37[48]$ & \\
$\mathrm{N}-\mathrm{H}\left(\mathrm{HCONH}_{2}\right)$ & $4.71[48]$ & \\
$\mathrm{C}-\mathrm{H}\left(\mathrm{H}_{3} \mathrm{CCHO}\right)$ & $3.9[48]$ & \\
$\mathrm{C}-\mathrm{O}\left(\mathrm{CH}_{2} \mathrm{O}\right)$ & $7.7[49]$ & \\
\hline
\end{tabular}

The thermodynamic values in this process $\left[E_{A}\left(\mathrm{NH}_{2}{ }^{-}\right)=\right.$ $0.77 \mathrm{eV}$ and $D(\mathrm{C}-\mathrm{N})=4.37 \mathrm{eV}$ ] indicate a threshold energy of $3.6 \mathrm{eV}$. This is well below the incident electron energy for the measured resonances. Another possible mechanism for the production of $\mathrm{NH}_{2}{ }^{-}$anions comes from the fragmentation of $\mathrm{HCO}$ via the aforementioned mechanism into neutral $\mathrm{H}$ and CO fragments:

$$
e^{-}+\mathrm{HCONH}_{2} \rightarrow\left(\mathrm{HCONH}_{2}\right)^{*-} \rightarrow \mathrm{H}+\mathrm{CO}+\mathrm{NH}_{2}{ }^{-} .
$$

The thermodynamic threshold for this process is $4.2 \mathrm{eV}$, given the $E_{A}$ of $\mathrm{NH}_{2}$ along with the standard heat of formation values of formamide, $\mathrm{H}, \mathrm{CO}$, and $\mathrm{NH}_{2}$ from Table II, which is also well below the incident electron energy for the observed resonance.

b. Momentum imaging. Momentum images of the $\mathrm{NH}_{2}{ }^{-}$ anion from DEA to formamide with incident electron energies between 5.3 and $6.8 \mathrm{eV}$ are provided in Fig. 1. These momentum images have a $\pi / 2$ rad conical slice selection gate, which allows us to project the 3D momentum distribution in 2D with a uniform volume in momentum space. The compact momentum distribution of $\mathrm{NH}_{2}{ }^{-}$was subject to small distortions in the imperfect spectrometer fields, which we have addressed in the calibration procedure and by exploiting the symmetry about the incident electron axis (which is along the $y$ axis).

The momentum images and accompanying angular plots for $\mathrm{NH}_{2}{ }^{-}$are shown in Fig. 1. We observe that the distribution is isotropic at the lower incident electron energies but builds up a maximum fragment yield at about $105^{\circ}$ from the incident electron direction axis in the 6.8-eV data. In addition to the experimental data, the right column of Fig. 1 shows the computed angular distributions for ${ }^{2} \mathrm{~A}^{\prime \prime}$ (black continuous line) and ${ }^{2} \mathrm{~A}^{\prime}$ (blue dot-dashed line) Feshbach resonances, calculated under the assumption that the axial-recoil approximation applies, i.e., that the dissociating bond does not rotate during fragmentation. While the ${ }^{2} \mathrm{~A}^{\prime \prime}$ resonance gives a predicted distribution that is consistent with the measured distributions that show the prevalence of a peak at $\sim 105^{\circ}$ at the higher end of the 5.3-6.8-eV energy range, the calculated distribution for the ${ }^{2} \mathrm{~A}^{\prime}$ resonance is highly nonisotropic and bears little (a)
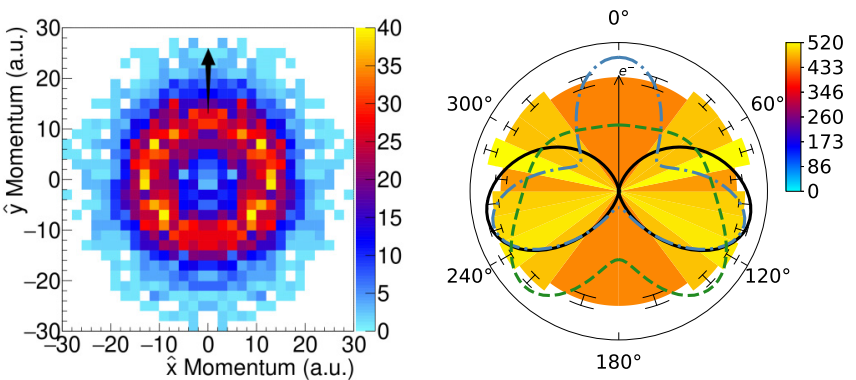

(b)
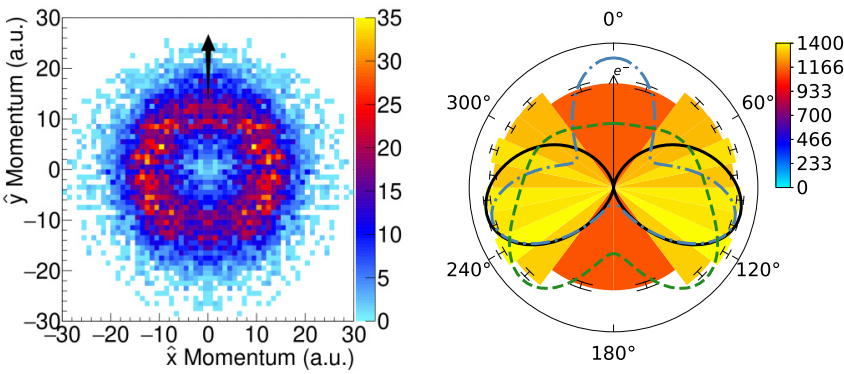

(c)
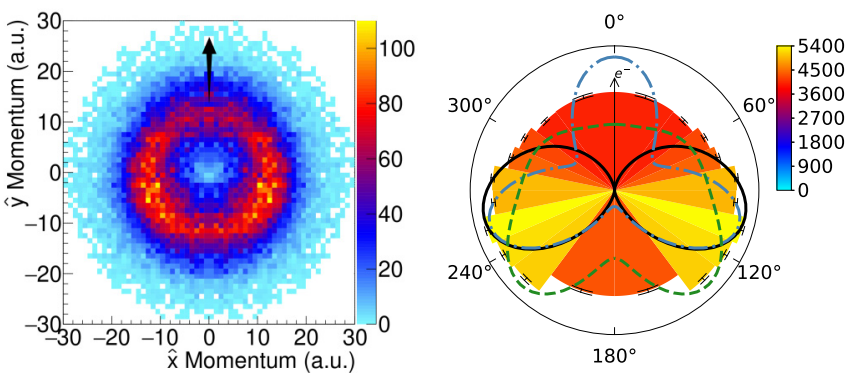

(d)
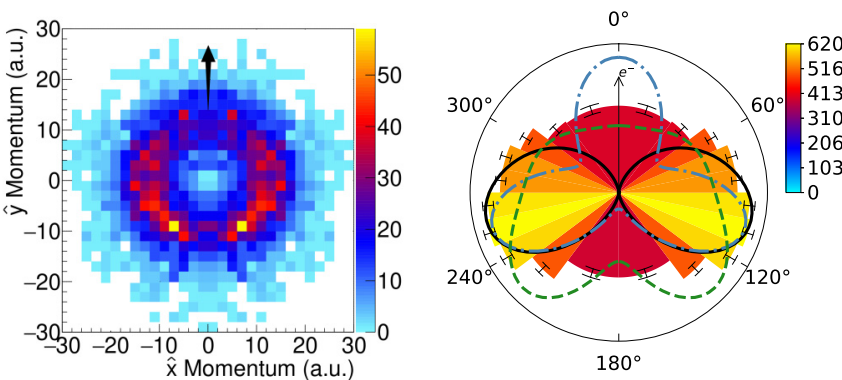

FIG. 1. Left: Momentum images of $\mathrm{NH}_{2}{ }^{-}$dissociation from DEA to formamide. Electron is incident in the $+y$ direction with energy (a) 5.3, (b) 5.8, (c) 6.3, and (d) $6.8 \mathrm{eV}$. Right: Histograms of dissociation angle of $\mathrm{NH}_{2}{ }^{-}$anions from formamide with incident electron energy of (a) 5.3 , (b) 5.8 , (c) 6.3 , and (d) $6.8 \mathrm{eV}$, and incident at $0^{\circ}$. The black line indicates the calculated angular distribution of $\mathrm{NH}_{2}{ }^{-}$for DEA to the lowest ${ }^{2} \mathrm{~A}^{\prime \prime}$ Feshbach resonance. The blue dotdashed line represents the calculated angular distribution of $\mathrm{NH}_{2}{ }^{-}$ for the ${ }^{2} \mathrm{~A}^{\prime}$ Feshbach resonance, and the green dashed line shows the ${ }^{2} \mathrm{~A}^{\prime}$ with a $30^{\circ}$ rotation of the recoil axis toward larger O-C-N angles.

resemblance with the measured distributions at any energy. This is consistent with a breakdown of axial recoil for this resonance pointing to an internal geometric change of the 


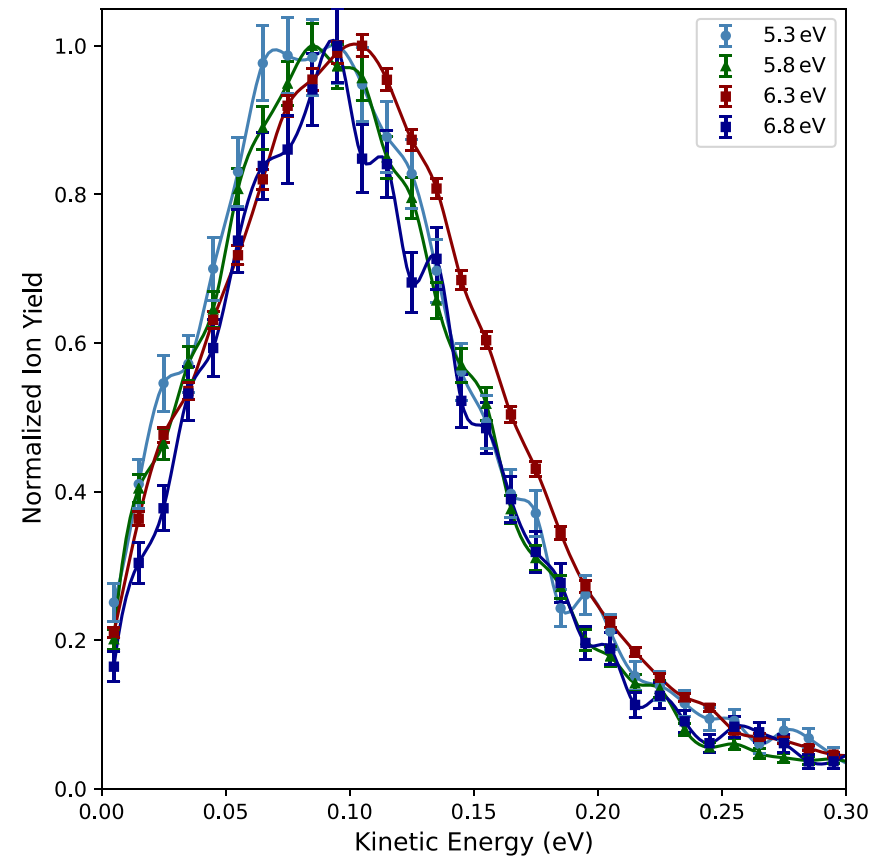

FIG. 2. Kinetic-energy spectra of $\mathrm{NH}_{2}{ }^{-}$fragment from DEA to formamide from incident electron energies $5.3-6.8 \mathrm{eV}$.

transient resonance anion prior to breakup. In an attempt to model this, we have also included the angular distribution of the ${ }^{2} \mathrm{~A}^{\prime}$ Feshbach resonance for a modified dissociation axis, which is modeled on a $30^{\circ}$ rotation of the $\mathrm{C}-\mathrm{N}$ dissociation axis towards larger $\mathrm{O}-\mathrm{C}-\mathrm{N}$ angle (green dashed line). This gives a more isotropic angular distribution that better agrees with the measured values at the lower end of the electron energy range, where the ${ }^{2} \mathrm{~A}^{\prime}$ resonance presumably dominates.

In Fig. 2 we provide the kinetic-energy distributions for the $\mathrm{NH}_{2}{ }^{-}$fragment for each electron energy. We find that the maximum of the $\mathrm{NH}_{2}{ }^{-}$distribution occurs at $\sim 0.09 \mathrm{eV}$. The kinetic-energy distribution does not change considerably with the incident energy over this range of incident energies. The peak at $\sim 0.09 \mathrm{eV}$ indicates a three-body dissociation, and/or significant rovibrational excitation in the molecular fragments, producing $\mathrm{NH}_{2}{ }^{-}$and $\mathrm{H}+\mathrm{CO}$ or $\mathrm{HCO}$ in the three-body or two-body dissociation, respectively. For the limiting case of a prompt three-body dissociation into cold molecular fragments, the neutral $\mathrm{H}$ atom could be released with as much as $1.6-3.1 \mathrm{eV}$ over this incident energy range. In the limiting case of two-body dissociation into rovibrationally hot molecular fragments, $1.6-3.1 \mathrm{eV}$ is the available internal energy in the excited $\mathrm{NH}_{2}{ }^{-}$and $\mathrm{HCO}$ fragments.

Previous mass-resolved anion fragment yield experiments [31,32] revealed two peaks producing $\mathrm{NH}_{2}{ }^{-}$at 5.9 and $6.8 \mathrm{eV}$. Despite the very small momentum available to the detected $\mathrm{NH}_{2}{ }^{-}$fragment, the subtle changes we find in the $\mathrm{NH}_{2}{ }^{-}$ momentum images and angular distributions between 5.9 and $6.8 \mathrm{eV}$, coupled with the theoretical predictions of two Feshbach resonances in this energy range, support the notion of two overlapping resonances contributing to the $\mathrm{NH}_{2}{ }^{-}$production. The $6.8-\mathrm{eV}$ resonance is expected to have the dominant contribution at the higher incident electron energies due to the higher $\mathrm{NH}_{2}{ }^{-}$yield previously observed at $6.8 \mathrm{eV}$, relative to the smaller peak that occurs on the low-energy shoulder around $5.9 \mathrm{eV}[31,32]$.

\section{B. $\mathrm{O}^{-}$resonance at $10.0-11.5 \mathrm{eV}$}

a. Pathways to formation. $\mathrm{O}^{-}$is one of the dominant fragments for incident electrons with energies between 10 and $11.5 \mathrm{eV}$ [32]. The formation of $\mathrm{O}^{-}$anions through DEA to formamide requires the cleaving of the $\mathrm{C}-\mathrm{O}$ double bond with a bond dissociation enthalpy of $7.7 \mathrm{eV}$. There are three possible two-body breakups of formamide and several feasible three-body processes. We will provide the two-body mechanisms here.

The two-body fragmentation that produces $\mathrm{O}^{-}$along with neutral aminomethylene $\left(\mathrm{HCNH}_{2}\right)$ is given by

$$
e^{-}+\mathrm{HCONH}_{2} \rightarrow\left(\mathrm{HCONH}_{2}\right)^{*-} \rightarrow \mathrm{HCNH}_{2}+\mathrm{O}^{-} .
$$

From the thermodynamic data in Table II, the threshold for this process is expected to be $6.2 \mathrm{eV}$. Additional two-body processes can be conceived by rearrangement of the hydrogen atoms in the neutral counterpart to $\mathrm{O}^{-}$. This includes

$$
e^{-}+\mathrm{HCONH}_{2} \rightarrow\left(\mathrm{HCONH}_{2}\right)^{*-} \rightarrow \mathrm{H}_{2} \mathrm{CNH}+\mathrm{O}^{-}
$$

and

$$
e^{-}+\mathrm{HCONH}_{2} \rightarrow\left(\mathrm{HCONH}_{2}\right)^{*-} \rightarrow \mathrm{CH}_{3} \mathrm{~N}+\mathrm{O}^{-} .
$$

These processes have thresholds of 4.2 and $6.3 \mathrm{eV}$, respectively (based on the values in Table II).

The three-body mechanism that produces $\mathrm{O}^{-}, \mathrm{HCN}$, and molecular hydrogen $\mathrm{H}_{2}$, due to two $\mathrm{N}-\mathrm{H}$ bonds and a C-O bond breaking, has a threshold of $4.4 \mathrm{eV}$, while the three-body mechanism producing $\mathrm{O}^{-}, \mathrm{HCN}$, and $\mathrm{H}_{2}$, by $\mathrm{C}-\mathrm{H}$ break, $\mathrm{N}-\mathrm{H}$ break, and C-O break, has a threshold of $5.0 \mathrm{eV}$. Consequently, these three-body processes may also play a role in the observed resonance between 10 and $11.5 \mathrm{eV}$.

b. Momentum imaging. The measured momentum distributions for the $\mathrm{O}^{-}$anion fragment from DEA to formamide are provided in Fig. 3. Here, we incorporated a $\pi / 2$-rad selection gate on the 3D momentum sphere. Note that our analysis of $\mathrm{O}^{-}$fragments did not impose cylindrical symmetry. This leads to momentum distributions that are slightly asymmetric about the incident electron direction axis, possibly due to minor imperfections in the electric fields within the spectrometer, small variations in detection efficiency across the face of the detector, and statistical uncertainties.

The $\mathrm{O}^{-}$momentum is sharply peaked at $\sim 120^{\circ}$ from the incident electron direction. The angular distributions of the $\mathrm{O}^{-}$anion fragment provided in Fig. 3 (right column) explicate this fact. This suggests that the $\mathrm{O}^{-}$fragments are ejected promptly along the $\mathrm{C} \rightarrow \mathrm{O}$ bond axis with little or no rotation of the C-O bond. The $120^{\circ}$ angle between the dissociating $\mathrm{C}-\mathrm{O}$ bond and the incident electron is consistent with a high electron attachment probability for incident electrons along the $\mathrm{C} \rightarrow \mathrm{N}$ direction. The $\mathrm{O}-\mathrm{C}-\mathrm{N}$ bond angle was calculated previously [50] for the equilibrium geometry of neutral formamide to be $\sim 125^{\circ}$.

The peak magnitude of the momentum distributions increases with the incident electron energy (from $\sim 10$ a.u. at $10.0 \mathrm{eV}$ to $\sim 25$ a.u. at $11.5 \mathrm{eV}$ ). The kinetic-energy spectra of $\mathrm{O}^{-}$anion for $10-11.5 \mathrm{eV}$ incident electrons are displayed in 
(a)
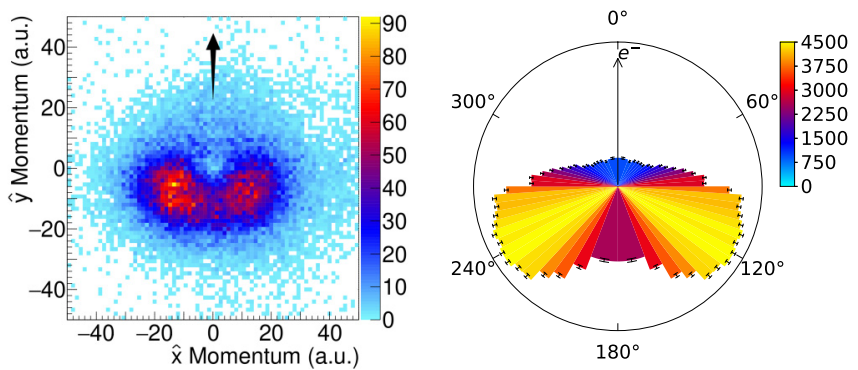

(b)
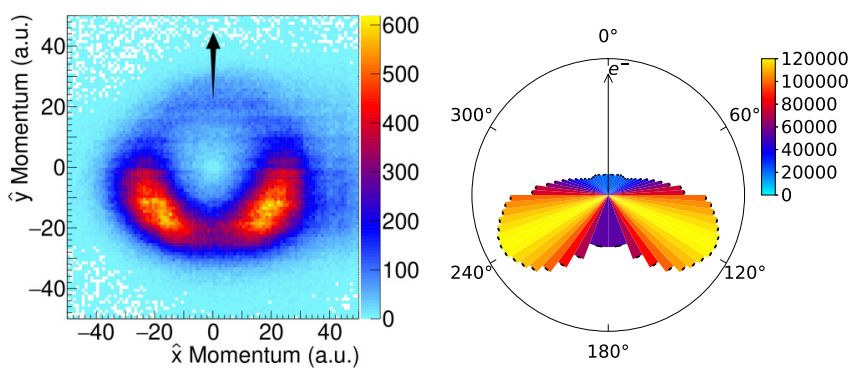

(c)
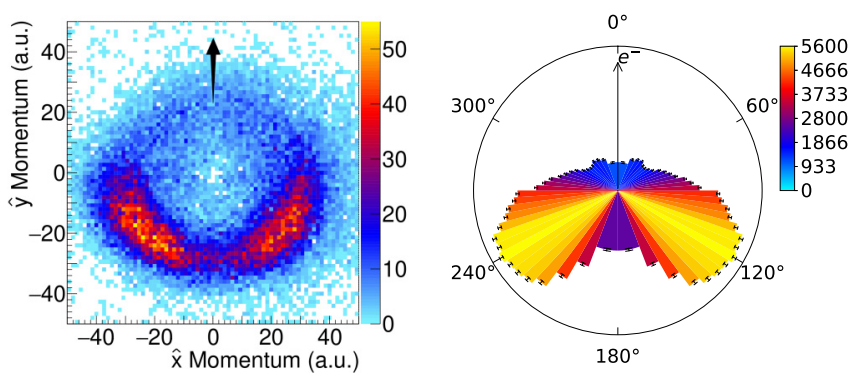

(d)
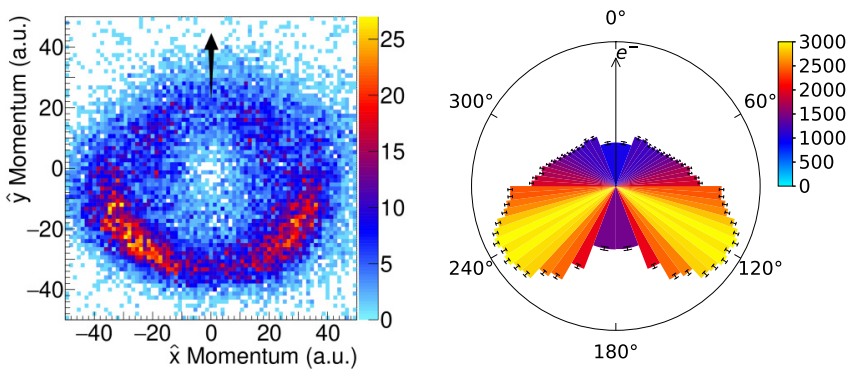

FIG. 3. Left: Momentum images of $\mathrm{O}^{-}$dissociation from DEA to formamide. Electron is incident in the $+y$ direction with energy (a) 10.0, (b) 10.5, (c) 11.0, and (d) $11.5 \mathrm{eV}$. Right: Histograms of dissociation angle of $\mathrm{O}^{-}$anions from formamide with incident electron energy of (a) 10.0, (b) 10.5 , (c) 11.0 , and (d) $11.5 \mathrm{eV}$ in the incident at $0^{\circ}$.

Fig. 4. The maxima of these kinetic-energy spectra increase approximately linearly with the incident electron energy. In addition to the formamide target gas, background gas in the target region includes a small but significant presence of water. As the incident electron energy increases, so does the production of $\mathrm{O}^{-}$anions from DEA to water [51]. While the impact of water background on the $\mathrm{O}^{-}$signal is small,

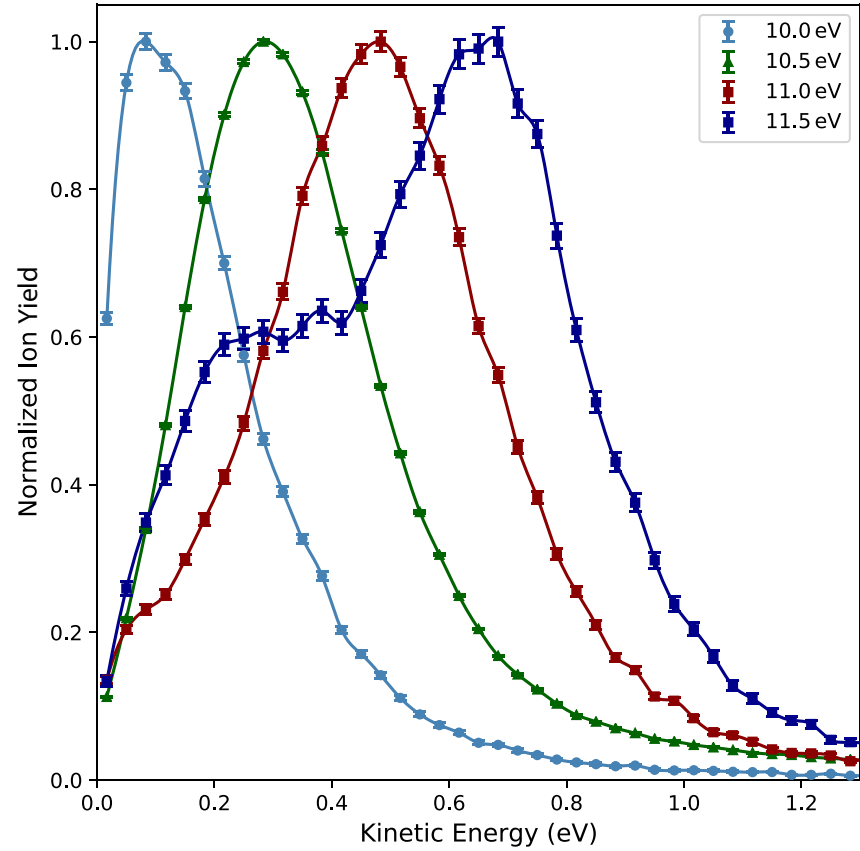

FIG. 4. Kinetic-energy spectra of $\mathrm{O}^{-}$fragment produced from DEA to formamide at incident electron energies $10.0-11.5 \mathrm{eV}$. The lower-energy peak of the double-peak structure for the 11.5-eV curve is attributed to $\mathrm{O}^{-}$from gaseous water within the interaction region.

we attribute the double-peak feature in the kinetic-energy distribution for the highest present incident electron energy of $11.5 \mathrm{eV}$ to water contamination in the interaction region. Additionally, the forward component (between $270^{\circ}$ and $90^{\circ}$ ) of the angular distribution starts to increase at $11.5 \mathrm{eV}$, which is also indicative of water contamination [13,36]. For the limiting case of two-body dissociation, the internal energy in the $\mathrm{HCNH}_{2}$ fragment, which may subsequently isomerize or undergo a secondary dissociation, is $3.6-4.2 \mathrm{eV}$.

\section{C. $\mathrm{H}^{-}$resonance at $10.0-11.5 \mathrm{eV}$}

The production of $\mathrm{H}^{-}$anions from DEA to formamide is known to have a peak yield at an incident electron energy of $6.5 \mathrm{eV}$ along with a less dominant peak at $10.5 \mathrm{eV}$ [32]. In the present experiments the substantial background of $\mathrm{H}^{-}$, due to the small but significant water contamination in the interaction region, produced ambiguous results around $6.5 \mathrm{eV}$ incident energies, so they are not presented here. At higher incident energies the much smaller DEA cross section for water reduces the $\mathrm{H}^{-}$contamination by approximately two orders of magnitude, so the present results for $\mathrm{H}^{-}$from formamide in the $10.0-11.5-\mathrm{eV}$ range of incident electron energies are essentially background-free.

a. Pathways to formation. The process of DEA to formamide resulting in the dissociation of $\mathrm{H}^{-}$via cleaving of an $\mathrm{N}-\mathrm{H}$ bond proceeds as

$$
e^{-}+\mathrm{HCONH}_{2} \rightarrow\left(\mathrm{HCONH}_{2}\right)^{*-} \rightarrow \mathrm{HCONH}+\mathrm{H}^{-} .
$$

Considering the bond dissociation enthalpy of the N-H bond $(4.71 \mathrm{eV})$ and the electron affinity of atomic hydrogen $(0.75 \mathrm{eV})$, we find that the threshold for this production 
(a)
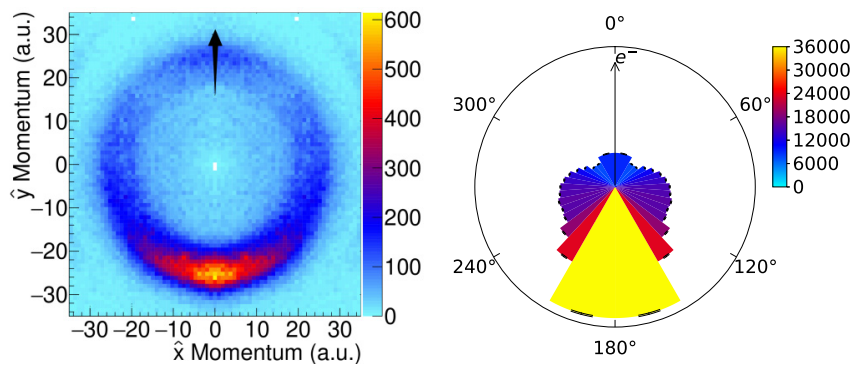

(b)
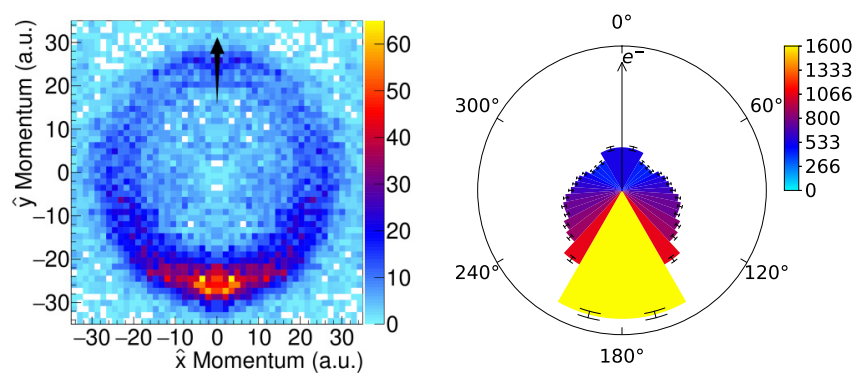

(c)
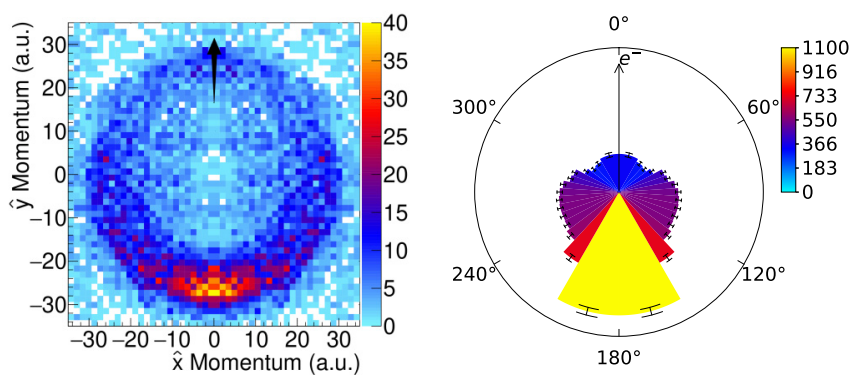

FIG. 5. Left: Momentum images of $\mathrm{H}^{-}$dissociation from DEA to formamide. Electron is incident in the $+y$ direction with energy (a) 10.5, (b) 11.0, and (c) $11.5 \mathrm{eV}$. Right: Histograms of dissociation angle of $\mathrm{H}^{-}$anions from formamide with incident electron energy of (a) 10.5, (b) 11.0 , and (c) $11.5 \mathrm{eV}$ in the direction of $0^{\circ}$.

mechanism is $3.9 \mathrm{eV}$, well below the incident electron energy for the observed resonance.

The C-H-bond-cleaving mechanism may proceed in two fashions, the first of which results in the production of a hydrogen anion and a single neutral counterpart:

$$
e^{-}+\mathrm{HCONH}_{2} \rightarrow\left(\mathrm{HCONH}_{2}\right)^{*-} \rightarrow \mathrm{CONH}_{2}+\mathrm{H}^{-} .
$$

Again, considering the electron affinity of atomic hydrogen and using the bond dissociation enthalpy for $\mathrm{C}-\mathrm{H}$ from acetaldehyde $\left(\mathrm{H}_{3} \mathrm{CCHO}\right)$, we find a threshold energy of $3.2 \mathrm{eV}$. The neutral $\mathrm{CONH}_{2}$ in the reaction above may dissociate further into neutral $\mathrm{CO}$ and $\mathrm{NH}_{2}$ molecular fragments, resulting in a third possible production mechanism for $\mathrm{H}^{-}$from formamide:

$$
e^{-}+\mathrm{HCONH}_{2} \rightarrow\left(\mathrm{HCONH}_{2}\right)^{*-} \rightarrow \mathrm{CO}+\mathrm{NH}_{2}+\mathrm{H}^{-} .
$$

Considering the standard heat of formation of formamide, $\mathrm{H}^{-}, \mathrm{CO}$, and $\mathrm{NH}_{2}$ from Table II, this process has a threshold of $4.3 \mathrm{eV}$. Thus both $\mathrm{C}-\mathrm{H}$-cleaving formation processes

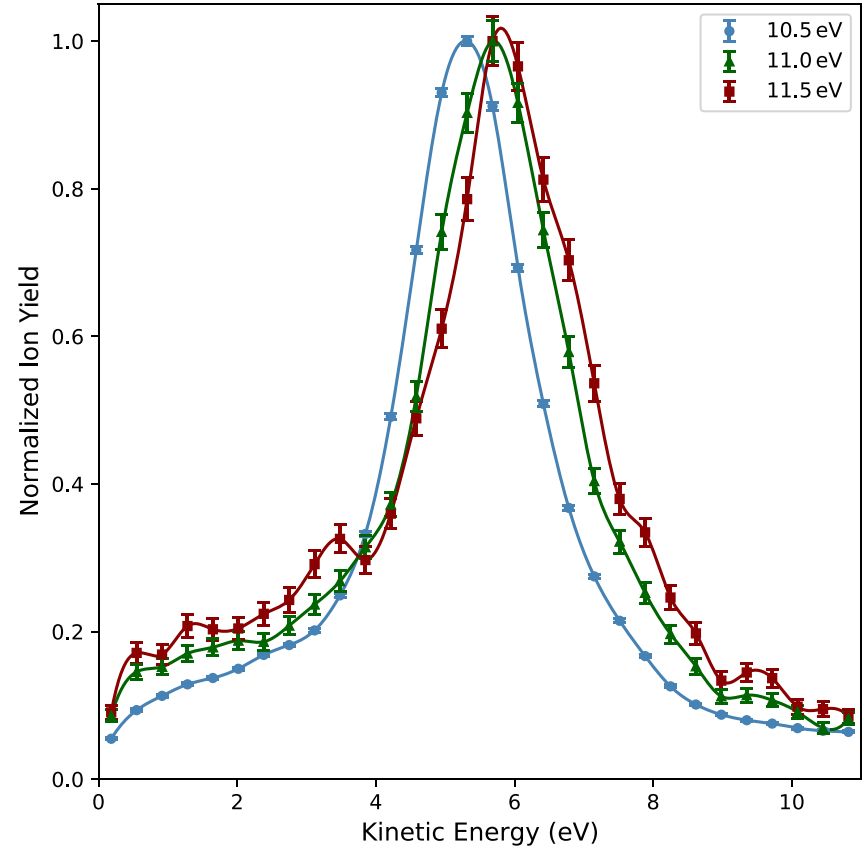

FIG. 6. Kinetic-energy spectra of $\mathrm{H}^{-}$fragment from DEA to formamide at incident electron energies $10.5-11.5 \mathrm{eV}$.

are achievable at these incident electron energies. While the formation at $\mathrm{H}^{-}$at the $\mathrm{C}$ site is possible, Hamann et al. [32] compared DEA on formamide to all its deuterated derivatives, and their findings suggest that DEA at these higher incident electron energies results in $\mathrm{H}^{-}$due to $\mathrm{N}-\mathrm{H}$ break without significant contributions of $\mathrm{H}^{-}$due to a C-H break.

b. Momentum imaging. The momentum images for the $\mathrm{H}^{-}$fragmentation channel are shown in Fig. 5 along with the respective angular distributions. Here, we incorporated a $\pi / 2$-rad selection gate on the 3D momentum sphere. In this fragmentation channel we observe that the $\mathrm{H}^{-}$anion strongly prefers to be emitted $\sim 180^{\circ}$ relative to the incident electron. Smaller features are also visible: there is a shoulder at $\sim 110^{\circ}$ and a small peak in the forward direction at $\sim 0^{\circ}$. This angular distribution appears unchanged over the range of $10.5-11.5 \mathrm{eV}$. As for the $\mathrm{O}^{-}$angular distributions, the sharp structures and high kinetic energy indicate that the dissociation is prompt and that little rotation of the dissociation axis occurs in the transient formamide anion prior to dissociation. The angular distribution is consistent with a high probability for electron attachment in the molecular frame of formamide along one $\mathrm{H} \rightarrow \mathrm{N}$ direction, and $\mathrm{H}^{-}$loss primarily from the same bond. It is conceivable that $\mathrm{H}^{-}$loss may occur, with a lower yield, from the other N-H bond. In the equilibrium geometry of formamide, the H-N-H bond angle was previously calculated to be $\sim 120^{\circ}$ [50], which could be a possible explanation of the shoulder at $\sim 110^{\circ}$. The kinetic-energy spectra of $\mathrm{H}^{-}$are shown in Fig. 6 and exhibit an increase in kinetic energy as the energy of the incident electrons increases. For the case of two-body dissociation, we can expect $1.2-1.7 \mathrm{eV}$ to be available as internal energy in the HCNH fragment, which may subsequently isomerize or undergo a secondary dissociation. 


\section{CONCLUSIONS}

We have presented the results of anion 3D momentum measurements of DEA to formamide, leading to $\mathrm{H}^{-}, \mathrm{O}^{-}$, and $\mathrm{NH}_{2}{ }^{-}$fragments in two energy regions, 5.3-6.8 eV and 10.0$11.5 \mathrm{eV}$. In the lower-energy region, where two resonances were reported previously $[31,32]$, the very low kinetic-energy distribution of $\mathrm{NH}_{2}{ }^{-}$does not change significantly between 5.3 and $6.8 \mathrm{eV}$. However, the angular distributions indicate a small but significant increase in anisotropy above $5.8 \mathrm{eV}$. $A b$ initio electron-scattering calculations of the electron attachment probability in the molecular frame for two Feshbach resonances offer insights into the character of the observed resonances. The qualitative agreement between the measured and calculated $\mathrm{NH}_{2}{ }^{-}$angular distributions for the 6.3- and 6.8-eV measurements is consistent with the dominant DEA process producing $\mathrm{NH}_{2}{ }^{-}$being due to electron attachment to the ${ }^{2} \mathrm{~A}^{\prime \prime}$ Feshbach resonance. The calculated ${ }^{2} \mathrm{~A}^{\prime}$ Feshbach resonance angular distribution is a poor match for the lower two experimental distributions at 5.3 and $5.8 \mathrm{eV}$. The ${ }^{2} \mathrm{~A}^{\prime}$ angular distribution for opening $\mathrm{O}-\mathrm{C}-\mathrm{N}$ angles is much more isotropic, and thus it is more qualitatively consistent with the present experimental results. In summary, we find that the two resonances that dissociate by $\mathrm{C}-\mathrm{N}$ break to form $\mathrm{NH}_{2}{ }^{-}$are not necessarily dipole-supported resonances, as recently reported [31]. The present experimental measurements and $a b$ initio electron-scattering calculations suggest that these are in fact ${ }^{2} \mathrm{~A}^{\prime \prime}$ and ${ }^{2} \mathrm{~A}^{\prime}$ Feshbach resonances with principal configurations $(\cdots)\left(2 \mathrm{a}^{\prime \prime}\right)^{1}\left(\sigma^{*}\right)^{2}$ and $(\cdots)\left(10 \mathrm{a}^{\prime}\right)^{1}\left(\sigma^{*}\right)^{2}$, respectively.

The anion resonances in the $10.0-11.5-\mathrm{eV}$ range are above the ionization threshold [52] of formamide. Electron- scattering calculations for resonances this high in the electronic continuum are highly challenging and beyond the scope of this work. Nevertheless, the sharp structures in the measured kinetic-energy and angular distributions for the $\mathrm{O}^{-}$and $\mathrm{H}^{-}$fragments provide information on the possible electron attachment and dissociation mechanisms. Within the assumption that the dissociation axis does not rotate significantly prior to dissociation, the angular distributions for $\mathrm{O}^{-}$and $\mathrm{H}^{-}$indicate that each product may be formed from a distinct resonance and that each of these two resonances has a distinct molecular-frame electron attachment probability.

\section{ACKNOWLEDGMENTS}

Work performed at the University of California Lawrence Berkeley National Laboratory was supported by the U.S. Department of Energy (DOE), Office of Science (SC), Division of Chemical Sciences of the Office of Basic Energy Sciences, under Contract No. DE-AC02-05CH11231 and by the DOE SC, Office of Workforce Development for Teachers and Scientists, Office of Science Graduate Student Research (SCGSR) program. The SCGSR program is administered by the Oak Ridge Institute for Science and Education for the DOE under Contract No. DE-SC0014664. Work performed at the University of Nevada, Reno, was supported by National Science Foundation Grant No. NSF-PHY-1807017. G.P. acknowledges support from the McNair Scholars Program at the University of Nevada, Reno. We are indebted to the RoentDek Company for long-term support with detector hardware and software.
[1] G. J. Schulz, J. Chem. Phys. 33, 1661 (1960).

[2] F. H. Dorman, J. Chem. Phys. 44, 3856 (1966).

[3] R. Compton and L. Christophorou, Phys. Rev. 154, 110 (1967).

[4] C. E. Melton, J. Chem. Phys. 57, 4218 (1972).

[5] S. Trajmar and R. Hall, J. Phys. B: At. Mol. Phys. 7, L458 (1974).

[6] C. E. Klots and R. N. Compton, J. Chem. Phys. 69, 1644 (1978).

[7] M. Jungen, J. Vogt, and V. Staemmler, Chem. Phys. 37, 49 (1979).

[8] M. Curtis and I. Walker, J. Chem. Soc., Faraday Trans. 88, 2805 (1992).

[9] W. Eck, V. Stadler, W. Geyer, M. Zharnikov, A. Gölzhäuser, and M. Grunze, Adv. Mater. 12, 805 (2000).

[10] P. M. Mendes, S. Jacke, K. Critchley, J. Plaza, Y. Chen, K. Nikitin, R. E. Palmer, J. A. Preece, S. D. Evans, and D. Fitzmaurice, Langmuir 20, 3766 (2004).

[11] O. Dulub, M. Batzilln, S. Solovev, E. Loginova, A. Alchagirov, T. E. Madey, and U. Diebold, Science 317, 1052 (2007).

[12] M.-M. Walz, M. Schirmer, F. Vollnhals, T. Lukasczyk, H.-P. Steinrück, and H. Marbach, Angew. Chem., Int. Ed. 49, 4669 (2010).

[13] L. G. Christophorou and J. K. Olthoff, Fundamental Electron Interactions with Plasma Processing Gases (Kluwer Academic/Plenum, New York, 2004).

[14] W. Morgan, in Fundamentals of Plasma Chemistry, Advances in Atomic, Molecular, and Optical Physics, edited by B. Bederson and H. Walther (Academic, San Diego, 2000), Vol. 43 pp. 79 110.

[15] B. Boudaiffa, P. Cloutier, D. Hunting, M. A. Huels, and L. Sanche, Science 287, 1658 (2000).

[16] X. Pan, P. Cloutier, D. Hunting, and L. Sanche, Phys. Rev. Lett. 90, 208102 (2003).

[17] H. Abdoul-Carime, S. Gohlke, and E. Illenberger, Phys. Rev. Lett. 92, 168103 (2004).

[18] S. Ptasińska and L. Sanche, Phys. Rev. E 75, 031915 (2007).

[19] L. Sanche, Mass Spectrom. Rev. 21, 349 (2002).

[20] J. M. Gingell, N. J. Mason, H. Zhao, I. C. Walker, and M. R. F. Siggle, Chem. Phys. 220, 191 (1997).

[21] M. H. F. Bettega, Phys. Rev. A 81, 062717 (2010).

[22] M. Vinodkumar, C. Limbachiya, H. Desai, and P. C. Vinodkumar, J. Appl. Phys. 116, 124702 (2014).

[23] M. G. P. Homem, I. Iga, G. L. C. de Souza, A. I. Zanelato, L. E. Machado, J. R. Ferraz, A. S. dos Santos, L. M. Brescansin, R. R. Lucchese, and M.-T. Lee, Phys. Rev. A 90, 062704 (2014).

[24] M. M. Dawley, C. Pirim, and T. M. Orlando, J. Phys. Chem. A 118, 1228 (2014).

[25] H. L. Barks, R. Buckley, G. A. Grieves, E. Di Mauro, N. V. Hud, and T. M. Orlando, ChemBioChem 11, 1240 (2010).

[26] R. Saladino, E. Carota, G. Botta, M. Kapralov, G. N. Timoshenko, A. Y. Rozanov, E. Krasavin, and E. Di Mauro, Proc. Natl. Acad. Sci. USA 112, E2746 (2015).

[27] B. Sivaraman, B. Nair, B. Raja Sekhar, N. Jones, S. Hoffmann, and N. Mason, Chem. Phys. Lett. 608, 404 (2014). 
[28] M. Ferus, P. Kubelík, and S. Civišs, J. Phys. Chem. A 115, 12132 (2011).

[29] T. P. M. Goumans, F. A. Gianturco, F. Sebastianelli, I. Baccarelli, and J. L. Rivail, J. Chem. Theory Comput. 5, 217 (2009).

[30] M. Seydou, A. Modelli, B. Lucas, K. Konate, C. Desfrançois, and J. P. Schermann, Eur. Phys. J. D 35, 199 (2005).

[31] Z. Li, M. Ryszka, M. M. Dawley, I. Carmichael, K. B. Bravaya, and S. Ptasińska, Phys. Rev. Lett. 122, 073002 (2019).

[32] T. Hamann, A. Edtbauer, F. Ferreira da Silva, S. Denifl, P. Scheier, and P. Swiderek, Phys. Chem. Chem. Phys. 13, 12305 (2011).

[33] E. Szymanska, B. G. Nair, N. J. Mason, and E. Krishnakumar, J. Phys.: Conf. Ser. 388, 052085 (2012).

[34] J. Fedor, Phys. Rev. Lett. 124, 199301 (2020).

[35] H. Adaniya, D. S. Slaughter, T. Osipov, T. Weber, and A. Belkacem, Rev. Sci. Instrum. 83, 023106 (2012).

[36] H. Adaniya, B. Rudek, T. Osipov, D. J. Haxton, T. Weber, T. N. Rescigno, C. W. McCurdy, and A. Belkacem, Phys. Rev. Lett. 103, 233201 (2009).

[37] D. S. Slaughter, T. Weber, A. Belkacem, C. S. Trevisan, R. R. Lucchese, C. W. McCurdy, and T. N. Rescigno, Phys. Chem. Chem. Phys. 22, 138983 (2020).

[38] T. N. Rescigno, B. H. Lengsfield III, and C. W. McCurdy, in Modern Electronic Structure Theory Part I, edited by D. R. Yarkony (World Scientific, Singapore, 1995), p. 501.

[39] D. S. Slaughter, A. Belkacem, C. W. McCurdy, T. N. Rescigno, and D. J. Haxton, J. Phys. B: At., Mol. Opt. Phys. 49, 222001 (2016).
[40] A. Bauder and H. H. Günthard, Helv. Chim. Acta 41, 670 (1958).

[41] M. A. Grela and A. J. Colussi, Int. J. Chem. Kinet. 20, 713 (1988).

[42] Data were retrieved from a database hosted by Argonne National Laboratory Active Thermochemical Tables (ATcT) based on ver. $1.122 \mathrm{p}$ of the Thermochemical Network, https://atct.anl. gov/Thermochemical\%20Data/version\%201.122p/index.php.

[43] M. W. Chase, Jr., NIST-JANAF Thermochemical Tables, 4th ed., doi: 10.18434/t42s31.

[44] C. T. Wickham-Jones, K. M. Ervin, G. B. Ellison, and W. C. Lineberger, J. Chem. Phys. 91, 2762 (1989).

[45] K. R. Lykke, K. K. Murray, and W. C. Lineberger, Phys. Rev. A 43, 6104 (1991).

[46] J. D. Cox, D. D. Wagman, and V. A. Medvedev, CODATA Key Values for Thermodynamics (Hemisphere, New York, 1989).

[47] S. J. Cavanagh, S. T. Gibson, M. N. Gale, C. J. Dedman, E. H. Roberts, and B. R. Lewis, Phys. Rev. A 76, 052708 (2007).

[48] Y.-R. Luo, Comprehensive Handbook of Chemical Bond Energies (CRC, Hoboken, 2007).

[49] B. Ruscic, J. Phys. Chem. A 119, 7810 (2015).

[50] G. Fogarasi and P. G. Szalay, J. Phys. Chem. A 101, 1400 (1997).

[51] J. Fedor, P. Cicman, B. Coupier, S. Feil, M. Winkler, K. Gluch, J. Husarik, D. Jaksch, B. Farizon, N. J. Mason, P. Scheier, and T. D. Märk, J. Phys. B: At., Mol. Opt. Phys. 39, 3935 (2006).

[52] R. Ballard, J. Jones, D. Read, A. Inchley, and M. Cranmer, Chem. Phys. Lett. 137, 125 (1987). 